\title{
Lixiviación de plata de minerales sulfurados argentíferos del complejo Marañón empleando el reactivo SANDIOSS
}

\section{SILVER LEACHING OF SILICATE SULFIDATED MINERALS FROM THE MARAÑÓN COMPLEX USING THE SANDIOSS REAGENT}

\author{
DANIEL F. LOVERA DÁVILA§*, BLANCA M. PERALTA DIAZ§, ENRIQUE C. CHANG \\ FRANCO§, VLADIMIR ARIAS
}

Recibido: 15 junio de 2018 / Aceptado: 04 agosto de 2018

§Instituto de Investigación-Facultad de Ingeniería Geológica, Minera, Metalúrgica y Geográfica-Universidad Nacional Mayor de San Marcos, Lima-Perú.

\section{Resumen}

Con el fin de estudiar los procesos metalúrgicos del mineral proveniente del complejo mineralógico Marañón con características polimetálicas, se acondiciono el mineral durante el proceso de chancado se usaron como agentes activadores y depresores el Bisulfito de Sodio $\left(\mathrm{NaHSO}_{3}\right)$, Cal, Sulfato de Zinc (ZnSO4), Cianuro de Sodio ( $\mathrm{NaCN}$ ) y se obtuvo una granulometría de 62\% -200 malla; en la flotación se obtuvo primero un bulk de $\mathrm{Cu}-\mathrm{Pb}$ usando MIBC como espumante, para luego obtener un concentrado de Zinc y obtener el relave de la flotación, el cual se llevó a una remolienda para obtener una granulometría óptima para la lixiviación, llegando a trabajar con un 96.5\% - 200 malla y pasando a lixiviar con Sandioss a dos concentraciones: 0.2 y $0.3 \%$ por un tiempo de 75 horas, titulando con Nitrato de Plata $\left(\mathrm{AgNO}_{3}\right)$ y usando de indicador Yoduro de Potasio (KI), llegando a obtener una recuperación del $71.9 \%$ de Ag a 0.2\% de Sandioss y $98.7 \%$ de Ag a 0.3\% de Sandioss.

Palabras clave: Lixiviación de Plata, recuperación de plata, sustituyente del cianuro, Sandioss

\begin{abstract}
In order to study the metallurgical processes of the mineral from the Marañón mineralogical complex with polymetallic characteristics, the mineral was conditioned during the crushing process. Sodium bisulphite (NaHSO 3$)$, Cal, Zinc Sulfate $\left(\mathrm{ZnSO}_{4}\right)$ were used as activating agents and depressants. ), Sodium cyanide $(\mathrm{NaCN})$ and a granulometry of $62 \%-200$ mesh was obtained; in the flotation, a bulk of $\mathrm{Cu}-\mathrm{Pb}$ was first obtained using MIBC as foaming agent, to obtain a Zinc concentrate and obtain the flotation tailings, which was taken to a regrind to obtain an optimum granulometry for leaching, reaching work with a $96.5 \%-200$ mesh and going to leach with Sandioss at two concentrations: 0.2 and $0.3 \%$ for a time of 75 hours, titrating with Silver Nitrate $\left(\mathrm{AgNO}_{3}\right)$ and using a Potassium Iodide $(\mathrm{KI})$ indicator, reaching obtain a recovery of $71.9 \%$ of $\mathrm{Ag}$ to $0.2 \%$ of Sandioss and $98.7 \%$ of Ag to $0.3 \%$ of Sandioss.
\end{abstract}

Key words: ilver leaching, silver recovery, cyanide substituent, Sandioss

Correspondencia de autor:

Instituto de Investigación-Facultad de Ingeniería Geológica, Minera, Metalúrgica y Geográfica-Universidad Nacional Mayorde San Marcos, Lima-Perú.

E-mail: dloverad@unmsm.edu.pe

\section{INTRODUCCIÓN}

El Perú goza un liderazgo en la minería mundial en la producción de minerales de Cobre, Zinc, Plomo, Plata, Oro, entre otros. En ese marco nos permite realizar investigaciones a los minerales sulfurados que contienen plata para su recuperación Hidrometalurgia. El presente estudio trata de encontrar una solución tecnológica a los 
minerales sulfurados del Complejo Marañón que tienen contenidos de Cobre, Zinc, Plomo y Plata según análisis químicos efectuados. Para ello debemos efectuar una buena caracterización del mineral de cabeza (Análisis: Químico, Mineralógico y Físico), para proceder posteriormente a desarrollar pruebas de Operaciones y Procesos Metalúrgicos (Chancado y Molienda, Flotación, Espesamiento, Filtración, Secado). Los productos obtenidos (Concentrado, Medios y Relaves) serán también caracterizados de manera similar al mineral de cabeza, para ubicar el grado de liberación y concentración de la Plata (Lovera et al., 2016; MINEM, 2017).

Los relaves son un conjunto de desechos de procesos mineros de concentración de minerales, estos arrastran trazas de los elementos valiosos con valor comercial en un muy bajo porcentaje, pero con el fin de maximizar ganancias las empresas de hoy en día siempre buscan recuperar estos metales pesados, aunque usando procedimientos o reactivos contaminantes (Arias, 2005; Sánchez, 2013; Araujo, 2014).

El Sandioss es un reactivo $100 \%$ lixiviante para minerales de oro y plata, tanto en óxidos como en sulfuros; relaves cianurados, relaves de amalgamación y escorias de fundición. Se aplica en pilas (heap leaching), en pozas (vat leaching) y tanques de agitación, obteniéndose alta recuperación mediante una lixiviación gradualmente sostenida y eficiente. Es de fácil manipulación y no requiere de condiciones especiales de compra, almacenamiento y transporte. Al ser un reactivo no contaminante y ofrecer una lixiviación óptima, se buscó implementarlo en la obtención de plata de un mineral proveniente del complejo Marañón- Región Huánuco, consistente en minerales metálicos de Cobre, Plata, Oro en esquistos, Níquel, Cobalto, Titanio, Platino, entre otros- y de esa forma sustituir al común y ampliamente usado $\mathrm{NaCN}$ durante la lixiviación. (Lovera, 2017; Habashi, 2006).

Al poseer el mineral un carácter polimetálico, las técnicas a emplearse indican deprimir o extraer los elementos que generen algún tipo de interferencia para de esa forma maximizar la extracción, de Ag en este caso, por ello en los procesos de concentración de minerales se extrajo el máximo posible de los metales valiosos, dejándonos así un relave listo para ser tratado y recuperar el máximo de plata posible (Misari,1993).

En el presente trabajo se utilizó el método de lixiviación mediante agitación, siguiendo principios básicos y aplicando el Sandioss de la misma forma y misma concentración que se aplicaría el $\mathrm{NaCN}$ en un ensayo estándar.

El reactivo Sandioss posee varios componentes entre los cuales se encuentran Ferrocianuros, los cuales hacen factible la lixiviación, a determinado porcentaje; al desconocer en un principio su concentración y como forma de corroborar los datos provenientes del análisis del reactivo se tuvo que determinar la concentración del Sandioss, para ello se hizo empleo el método gráfico quedando así establecido los gramos de Sandioss a disponer para $0.2 \%$ y $0.3 \%$ (Lovera, 2017).

\section{MATERIALES Y MÉTODOS}

Una vez transportado el mineral desde el complejo Marañón, se procedió a realizar su preparación mecánica, empleando una chancadora de quijada y una chancadora de cono y un molino de bolas. Con el objetivo de llevar el mineral a una granulometría de 100\% -10 um malla se pasó por la pulverizadora (Figura 1) después de las chancadoras y una vez obtenida esta granulometría se llevó al molino de bolas para proceder con la molienda 
(Azañero, 1987).

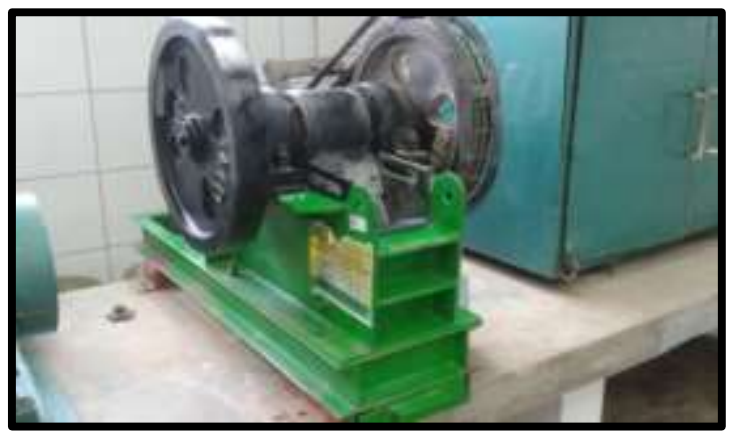

Figura 1. Chancadora Primaria

Para la molienda se trabajó con una relación liquido-solido (L/S) de 1/2, siendo $1 \mathrm{~kg}$ de mineral por $500 \mathrm{ml}$ de agua. Se buscó deprimir ciertos metales, por ello se acondicionó el molino agregando $\mathrm{NaCN} 1 \%$, Cal 0.4 g, ZnSO4 (10\%) y NaHSO3 (10\%).

El tiempo empleado para la molienda se determinó por medio de un ensayo previo de moliendabilidad, empleando la misma relación L/S y la misma cantidad de mineral por cada medida, tomando así 4 medidas para los tiempos de 5, 10, 15 y 20 minutos.

Una vez establecido el tiempo de 12 minutos para obtener una granulometría del $62 \%$ 200 malla, se procedió a llevar el mineral a una celda de flotación para continuar con el proceso de concentración del mineral, en el cual se buscó primero extraer el cobre y el plomo.

Para poder obtener el bulk $\mathrm{Cu}-\mathrm{Pb}$ se acondicionó la celda por unos 5 minutos a 1200 RPM con Cal (hasta que se alcance un pH de 7.3) $\mathrm{NaCN}$ (1\%), $\mathrm{ZnSO}_{4}(10 \%), \mathrm{Z11}(1 \%)$ y espumante MIBC (5 gotas). Una vez concluyo el tiempo de acondicionamiento se comenzó a insuflar el aire a la celda y se flotó por 10 minutos, concluyendo con ello la extracción de Cobre y Plomo (Figura 2).

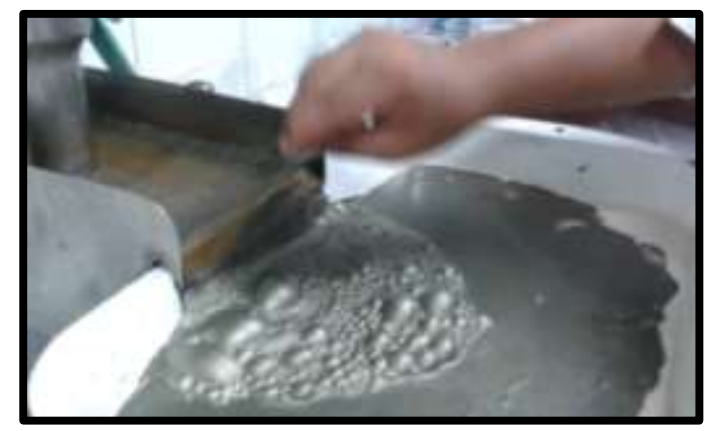

Figura $\mathrm{N}^{\circ}$ 2. Obtención del Bulk $\mathrm{Cu}-\mathrm{Pb}$

Una vez que se extrajo el bulk se procedió a extraer el Zinc, para ello se acondicionó la celda por 5 minutos a $1200 \mathrm{RPM}$ usando $\mathrm{Cal}$ (hasta que el $\mathrm{pH}$ aumente a 11.3), $\mathrm{NaCN}$ (1\%), CuSO4 (10\%), Z11 (1\%) y espumante MIBC (5 gotas). Después del tiempo de acondicionamiento se floto por unos 10 minutos y una vez extraído el Zinc se procedió a 
lavar la celda y recuperar el relave (Figura 3).

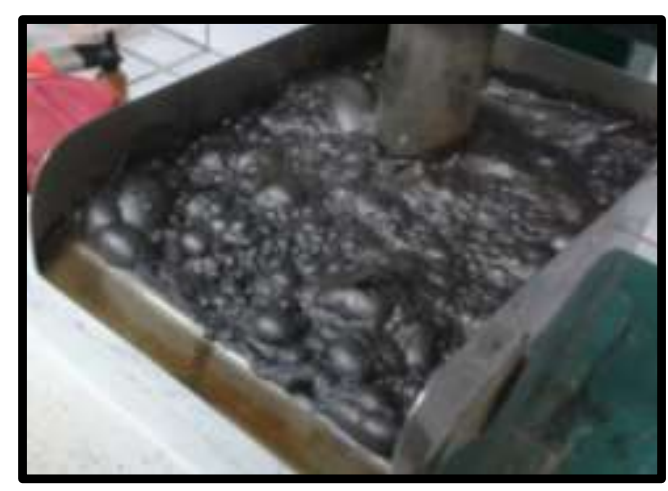

Figura 3. Obtención del Zinc en la celda de flotación

Posterior al lavado de la celda, se llevaron los concentrados y el relave a un secado usando un filtro al vacío con un compresor de aire a unos 50 PSI, luego, acabado el secado en filtro, se llevaron los concentrados y el relave (tortas) al horno por unos 7 minutos a unos $100{ }^{\circ} \mathrm{C}$, logrando así evaporar por completo el agua (Figura 4).

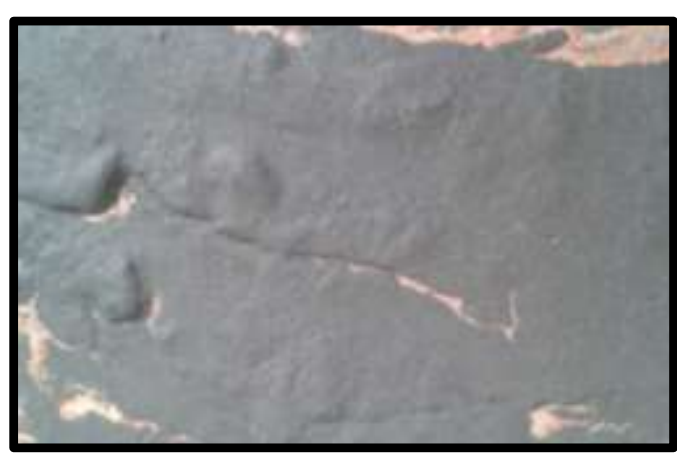

Figura $\mathrm{N}^{\circ}$ 4. Mineral de $\mathrm{Zn}$ seco

Luego del secado se sacó el balance de materia (Tabla 1), dándonos que por cada kg de mineral procesado obteníamos 706 gr de relave con una recuperación total de 993 g de mineral.

Tabla 1. Balance de materia de Flotación por 1kg de mineral

\begin{tabular}{lc}
\hline \multicolumn{1}{c}{ Productos } & Peso $(\mathbf{g})$ \\
\hline Concentrado de Cobre & 119 \\
Concentrado de Zinc & 168 \\
Relave & 706 \\
Total & 993 \\
\hline
\end{tabular}

Para una buena lixiviación en pruebas de laboratorio se suelen manejar granulometrías inferiores a 90\% -200malla, para ello se pasó el relave por un tamizado en húmedo en una malla 200 y un posterior secado para calcular su granulometría, la cual nos dio 74\% (200 um malla). Siendo esta no óptima se realizó una remolienda haciendo otra prueba de moliendabilidad calculando para varios tiempos la granulometría obtenida (Tabla 2). 
Tabla 2. Ensayo de moliendabilidad para la remolienda

\begin{tabular}{cc}
\hline Tiempo & M-200 \\
\hline 10 & 82.692 \\
15 & 95.2 \\
20 & 98.176 \\
25 & 98.806 \\
\hline
\end{tabular}

Una vez dada la remolienda por 16 minutos se obtuvo una granulometría de $96.5 \%$ 200malla siendo apropiada para lixiviar (Figura 5).

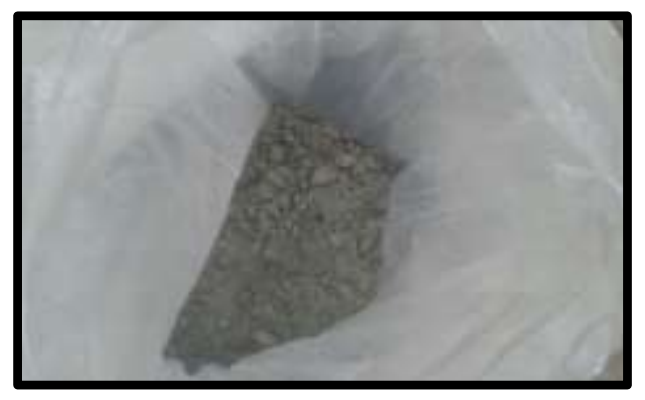

Figura 5. Reactivo Sandioss

Al trabajar con el reactivo Sandioss se tuvo que tomar en cuenta la variedad de sus componentes, entre ellos los principales son los ferrocianuros que hacen posible una lixiviación, por lo mismo se mandó a analizar el Sandioss a utilizar y con los componentes definidos se realizó unas pruebas preliminares y se aplicó el método gráfico para calcular los gramos necesarios de Sandioss para llegar a los porcentajes a trabajar (0.2 y $0.3 \%$ ).

Para poder lixiviar es necesario contar con un titulante y un indicador, por ello se elaboró 1 litro de Nitrato de Plata a $4.33 \mathrm{~g} / \mathrm{L}$ como la solución titulante, teniendo los cuidados necesarios con su almacenado, y Yoduro de Potasio como indicador para la plata por su viraje amarillento.

Se procedió a lixiviar en vasos de precipitado Pirex en agitadores magnéticos por 75 horas a las concentraciones de $0.2 \%$ y $0.3 \%$ de Sandioss, trabajando a un $\mathrm{pH}$ superior a 11.5 y 800 RPM a temperatura ambiente. La determinación de la extracción de plata se dio tomando alícuotas de $15 \mathrm{ml}$ a diferentes tiempos, titulando $5 \mathrm{ml}$, almacenando muestras de $10 \mathrm{ml}$ y reponiendo la cantidad de Sandioss y agua consumidas después de cada medición. El cálculo en ppm de la cantidad de plata presente en las muestras se realizó con equipos de espectrofotometría.

Antes de extraer la plata presente en el relave del mineral se procedió a prepararlo mecánicamente para obtener una granulometría adecuada y concentrarlo para obtener solo el relave. Se trajeron $50 \mathrm{~kg}$ de mineral de cabeza desde el complejo Marañón, el cual fue analizado previamente por difracción de rayos $X$ (Tabla $\mathrm{N}^{\circ} 3$ ), determinando una ley de plata de 386.79 g/TM (Lovera, 2005; Quiñones, 2009, 2010; Landauro, 2010) 
Tabla 3. Análisis del mineral de cabeza

\begin{tabular}{ccc}
\hline Elemento & Símbolo & Cantidad \\
\hline Cobre & $\mathrm{Cu}$ & $4.05 \%$ \\
Zinc & $\mathrm{Zn}$ & $8.95 \%$ \\
Plomo & $\mathrm{Pb}$ & $0.90 \%$ \\
Hierro & $\mathrm{Fe}$ & $25.40 \%$ \\
plata & $\mathrm{Ag}$ & $386.79 \mathrm{gr} / \mathrm{t}$ \\
\hline
\end{tabular}

Del análisis obtenido se pudo observar que el mineral posee una ley media de cobre, zinc, plomo, hierro y plata, siendo comercialmente viable su procesamiento y la obtención de estos, quedando así demostrada la naturaleza polimetálica del mineral y del yacimiento del cual se extrajo.

Luego del análisis se procedió a chancar y moler el mineral usando la curva de moliendabilidad obtenida con las pruebas preliminares hechas con el mineral en el molino de bolas (Figura 6).

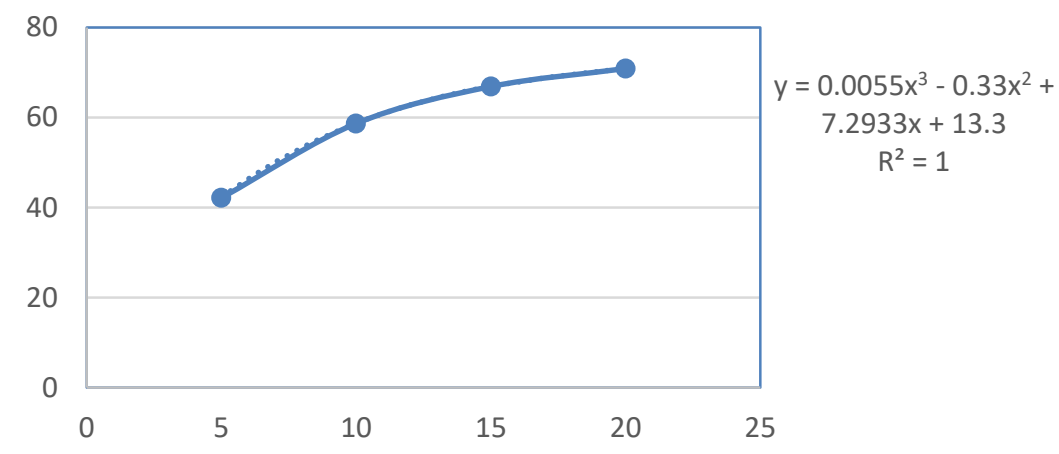

Figura $\mathrm{N}^{\circ}$ 6. Gráfico del ensayo de moliendabilidad

Para la gráfica de moliendabilidad se usó la línea de tendencia con mayor aproximación a la gráfica real, dándonos una ecuación cubica y obteniendo así el tiempo optimo; un detalle que se observó conforme avanzaba el proyecto es el hecho que la molienda no es uniforme, es decir, al ir realizando más moliendas la granulometría obtenida fue variando, por ello al concluir todas las moliendas se acumularon las pulpas y se obtuvo un aglomerado con una granulometría de $62.5 \%$ (200 um malla).

Al tener un porcentaje de plomo del $0.9 \%$ se debió extraer este mismo en un bulk junto con el cobre usando principalmente ZnSO4 como depresor de Zinc, Z11 como colector y MIBC. La cantidad de concentrado obtenido varió en la mayoría de pruebas de flotación, pero siempre manteniéndose entre los 117-122 g y dando al final una pérdida de 6 a $8 \mathrm{~g}$ de mineral por $\mathrm{kg}$ de mineral flotado, medidos por balanza electrónica. Ya extraído el bulk $\mathrm{Cu}-\mathrm{Pb}$ extrajimos el Zinc, usando el CuSO4 como depresor de Cobre, Z11 de colector y MIBC; al flotar el Zinc se observó su naturaleza marmatítica y la cantidad de concentrado obtenido variaba entre los 166-173 g por $\mathrm{kg}$ de mineral procesado, con una perdida entre $\operatorname{los} 5-8 \mathrm{~g}$. 
Para el secado del concentrado y el relave se inició usando el horno por unos $25 \mathrm{~min}$, pero dado el contenido de azufre, arsénico y antimonio presentes aun en baja concentración en el mineral se requirió el uso de un secado con filtro al vacío como paso inicial y un posterior secado en horno, mejorando el tiempo requerido y evitando la propagación de gases contaminantes. El secado por filtro requirió de $200 \mathrm{~mL}$ de agua adicionales para el lavado de la fuente y se empleó un compresor de aire de pistó CBS serie XV de 1HP.

Luego del tratamiento térmico la granulometría del material seco fue calculada realizando un tamizado en húmedo en malla 200, dejándonos una granulometría promedio de 73.8\% a -200; para pruebas de laboratorio es un estándar trabajar a tamaños de partícula inferiores a 90\% -200malla, a propósito de ello se hicieron pruebas de molienda para obtener un nuevo ensayo de moliendabilidad para la remolienda, los cuales se observan en la (Figura 7).

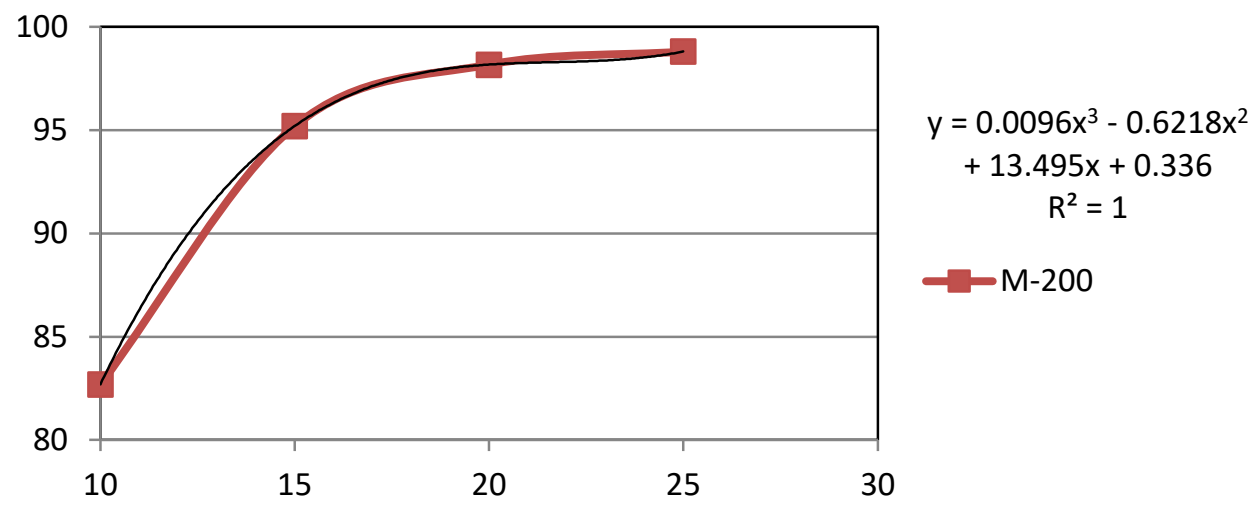

Figura 7. Gráfico de moliendabilidad para la remolienda

Para lixiviar se buscó el tiempo para obtener un $95 \%$ pasante en malla 200, sin embargo, por falta de calibración del molino empleado se llegó a un $96.5 \%$ pasante, siendo este valor resultante igual de óptimo para realizar una lixiviación.

Previa lixiviación, se elaboró la Figura 8 de la concentración de sandios mediante pruebas preliminares y se realizó el ajuste lineal dándonos la ecuación respectiva como se puede apreciar en la Tabla 4.

Tabla 4. Pruebas para determinar la concentración de Sandioss

\begin{tabular}{cc}
\hline Tiempo & Concentración \\
\hline 2 & 0.05 \\
4 & 0.01 \\
6 & 0.081 \\
8 & 0.113 \\
10 & 0.145 \\
12 & 0.175 \\
14 & 0.21 \\
16 & 0.23 \\
\hline
\end{tabular}




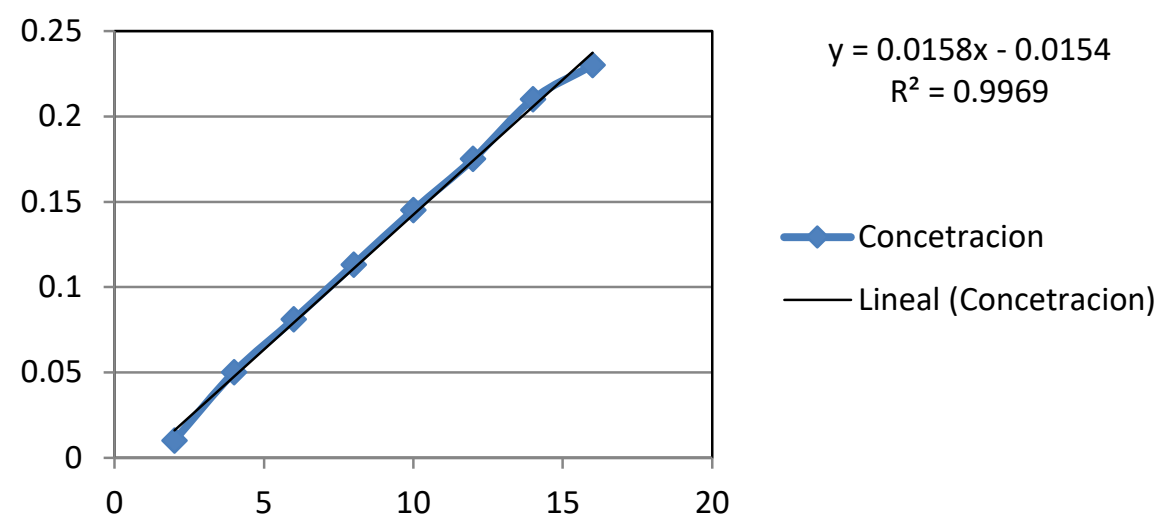

Figura 8. Gráfica de Concentración de Sandioss

A partir de ello lixiviamos por 75 horas para las concentraciones de Sandioss al 0.2 y $0.3 \%$, empleando un medidor de $\mathrm{pH}$ electrónico para controlar que este no ande por debajo de los 10.5 de $\mathrm{pH}$ ni superior a los 12.5; se emplearon agitadores magnéticos con medidor de temperatura y se regulo las revoluciones siempre constantes en 800 RPM a temperatura ambiente, lixiviando a esas condiciones $200 \mathrm{~g}$ de mineral en $400 \mathrm{ml} \mathrm{de} \mathrm{H}_{2} \mathrm{O}$ y titulando con AgNO3 preparado al $0.43 \%$ en 11 y de indicador KI (Mantell,1980; Morales, 2010; Mantell,1980; Morales, 2010).

La Tabla 5 muestran los datos del mineral y los resultados para el Sandioss al $0.2 \%$.

Tabla 5. Detalles del mineral y la lixiviación al 0.2\% de Sandioss

\begin{tabular}{lc}
\hline \multicolumn{2}{c}{ Metalurgia } \\
\hline DETALLES & Cantidad \\
\hline Peso seco de la muestra $(\mathrm{Kg})$ & 0.20 \\
Peso del agua $(\mathrm{Kg})$ & 0.40 \\
Finos en cabeza: Ag $(\mathrm{mg})$ & 14.4 \\
Cabeza Ensayada de Ag $(\mathrm{g} / \mathrm{TM})$ & 72.0 \\
Extracción $\mathrm{Ag}(\%)$ & 71.9 \\
\hline
\end{tabular}

Tabla 6. Resultados de la lixiviación de $0.2 \%$ de Sandioss

\begin{tabular}{|c|c|c|c|c|c|c|c|}
\hline \multirow{2}{*}{$\begin{array}{c}\text { TIEMPO } \\
\text { AGITACION } \\
\text { (h) }\end{array}$} & \multirow{2}{*}{$\begin{array}{l}\text { ALICUOTA } \\
(\mathrm{ml})\end{array}$} & \multicolumn{2}{|c|}{ pH } & \multirow{2}{*}{$\begin{array}{c}\text { LEY } \\
\text { PREGNANT } \\
(\mathbf{p p m}) \\
\mathbf{A g}\end{array}$} & \multirow{2}{*}{$\begin{array}{c}\begin{array}{c}\text { mg } \\
\text { descontados }\end{array} \\
\mathbf{A g}\end{array}$} & \multirow{2}{*}{$\begin{array}{c}\text { CONTENIDO } \\
\text { FINOS (mg) } \\
\mathrm{Ag}\end{array}$} & \multirow{2}{*}{$\begin{array}{c}\begin{array}{c}\text { EXTRACCION } \\
(\%)\end{array} \\
\mathbf{A g}\end{array}$} \\
\hline & & INICIAL & FINAL & & & & \\
\hline $\mathbf{0}$ & 0 & 3.91 & 11.54 & 0.000 & & 0.00 & 0.0 \\
\hline 2 & 15 & 12.02 & 12.02 & 5.93 & 0.089 & 2.37 & 16.5 \\
\hline 4 & 15 & 12.02 & 12.02 & 13.13 & 0.197 & 5.34 & 37.1 \\
\hline 6 & 15 & 12.04 & 12.04 & 19.01 & 0.285 & 7.89 & 54.8 \\
\hline 8 & 15 & 12.05 & 12.05 & 19.8 & 0.297 & 8.49 & 59.0 \\
\hline 24 & 15 & 11.81 & 11.81 & 21.54 & 0.323 & 9.48 & 65.9 \\
\hline 48 & 15 & 11.41 & 11.41 & 21.57 & 0.324 & 9.82 & 68.2 \\
\hline 75 & & 11.40 & 11.40 & 22.11 & 0.000 & 10.36 & 71.9 \\
\hline TOTAL & & & & & 1.515 & 10.36 & 71.94 \\
\hline
\end{tabular}


Como se puede apreciar de la Tabla 6 se trabajó tomando alícuotas de $15 \mathrm{ml}$, de las cuales $10 \mathrm{ml}$ son de muestra, analizadas por un equipo de espectrofotometría de absorbancia, y $5 \mathrm{ml}$ pasan a ser titulados con el nitrato; el $\mathrm{pH}$ no varió por fuera del rango, por ello no fue necesario el uso de cal para regular el pH y también se observa cómo fue creciendo la ley pregnant de la plata conforme aumentaba el tiempo de lixiviación, hasta llegar a una extracción del $71.94 \%$ en relación al contenido en finos de plata del mineral de cabeza.

A continuación, se presentan las Figuras $\mathrm{N}^{\circ} 9$ y 10 del crecimiento y evolución de la Ley Pregnant, solo con la finalidad de ver si hubo algún abrupto durante el proceso, y la cinética de extracción de valores metálicos para el Sandioss al $0.2 \%$.

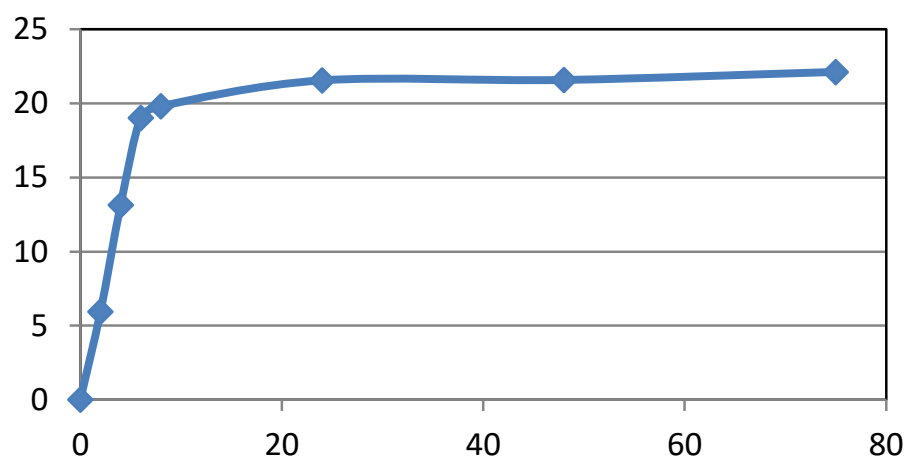

Figura 9. Ley Pregnant Ag 0.2\%

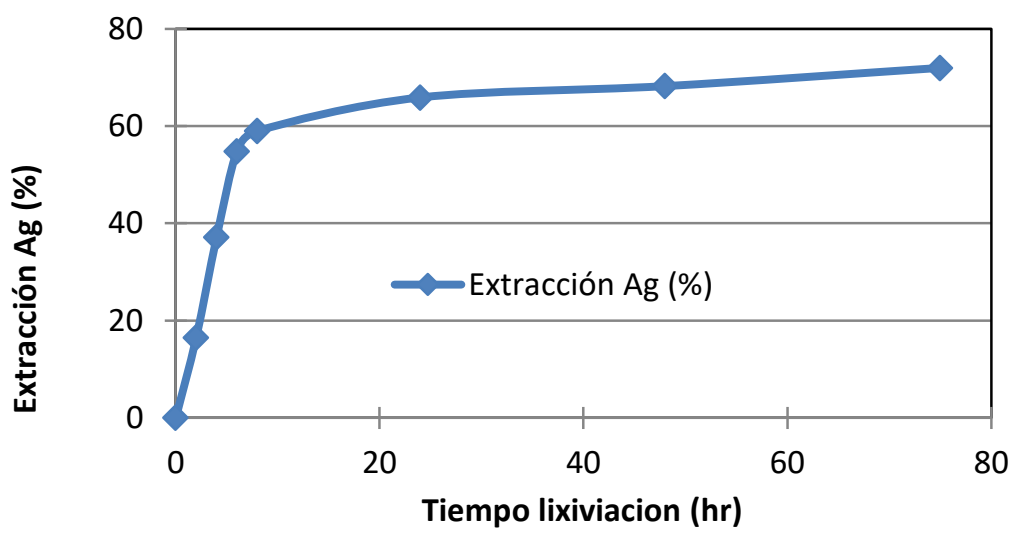

Figura 10. Cinética de Extracción de Valores metálicos Ag 0.2\%

Tabla 7. Resultados del proceso de Lixiviación

\begin{tabular}{lc}
\hline DETALLES & Cantidad \\
\hline Peso seco de la muestra $(\mathrm{Kg})$ & 0.20 \\
Peso del agua $(\mathrm{Kg})$ & 0.40 \\
Finos en cabeza: $\mathrm{Ag}(\mathrm{mg})$ & 14.4 \\
Cabeza ensayada de $\mathrm{Ag}(\mathrm{g} / \mathrm{TM})$ & 72.0 \\
Extracción $\mathrm{Ag}(\%)$ & 98.7 \\
\hline
\end{tabular}


Tabla 8. Detalles del mineral y la lixiviación al 0.3\%

\begin{tabular}{|c|c|c|c|c|c|c|c|}
\hline \multirow{2}{*}{$\begin{array}{c}\text { TIEMPO } \\
\text { AGITACION } \\
\text { (h) }\end{array}$} & \multirow{2}{*}{$\begin{array}{l}\text { ALICUOTA } \\
(\mathrm{ml})\end{array}$} & \multicolumn{2}{|c|}{ pH } & \multirow{2}{*}{$\begin{array}{c}\text { LEY } \\
\text { PREGNANT } \\
(\mathbf{p p m})\end{array}$} & \multirow{2}{*}{$\begin{array}{c}\begin{array}{c}\text { mg } \\
\text { descontados }\end{array} \\
\mathbf{A g}\end{array}$} & \multirow{2}{*}{$\begin{array}{c}\text { CONTENIDO } \\
\text { FINOS (mg) } \\
\mathbf{A g}\end{array}$} & \multirow{2}{*}{$\begin{array}{c}\text { EXTRACCION } \\
(\%) \\
\mathbf{A g}\end{array}$} \\
\hline & & $\begin{array}{c}\text { Ph } \\
\text { INICIAL }\end{array}$ & $\begin{array}{c}\text { pH } \\
\text { FINAL }\end{array}$ & & & & \\
\hline $\mathbf{0}$ & 0 & 3.52 & 11.42 & 0.000 & 0.000 & 0.00 & 0.0 \\
\hline 2 & 15 & 11.99 & 11.99 & 13.9 & 0.209 & 5.56 & 38.6 \\
\hline 4 & 15 & 12.12 & 12.12 & 17.33 & 0.260 & 7.14 & 49.6 \\
\hline 6 & 15 & 12.07 & 12.07 & 20.09 & 0.301 & 8.50 & 59.1 \\
\hline 8 & 15 & 12.07 & 12.07 & 21.54 & 0.323 & 9.39 & 65.2 \\
\hline 24 & 15 & 12.02 & 12.02 & 28.01 & 0.420 & 12.30 & 85.4 \\
\hline 48 & 15 & 11.51 & 11.51 & 29.58 & 0.444 & 13.35 & 92.7 \\
\hline 75 & & 11.50 & 11.50 & 30.65 & 0.000 & 14.34 & 98.7 \\
\hline TOTAL & & & & & 1.957 & 14.34 & 98.7 \\
\hline
\end{tabular}

Por lo apreciado en las Figuras 9 y 10 la extracción en las últimas mediciones tuvo un crecimiento lento, esto puede deberse a que la concentración usada extrae hasta ese máximo o podría necesitarse de otras condiciones especiales para elevar su extracción a esa concentración. Para el Sandioss al $0.3 \%$ se obtuvo estos resultados.

De acuerdo a las Tablas 7 y 8 se puede evidenciar que no fue necesaria la cal durante ningún momento del proceso al $0.3 \%$ de Sandioss ya que el $\mathrm{pH}$ se mantuvo constante después de cada reposición de Sandioss y agua en cada medición de parámetros, y que la cantidad de mg descontados se puede considerar como mínima dado que llegó a un total de $1.957 \mathrm{mg}$. A partir de estos resultados se construyeron las Figuras 11 y 12.

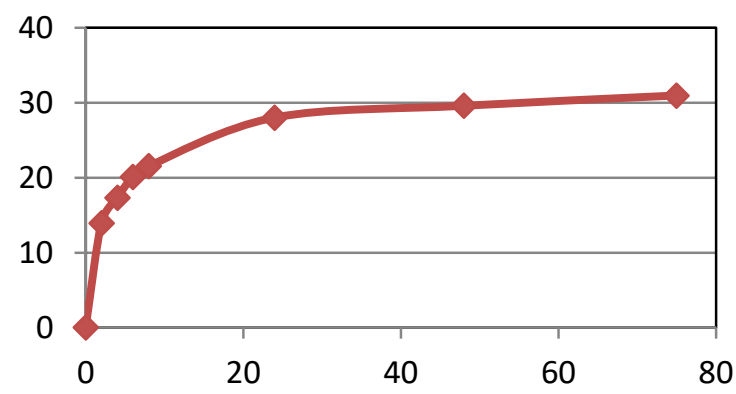

Figura 11. Ley Pregnant Ag 0.3\%

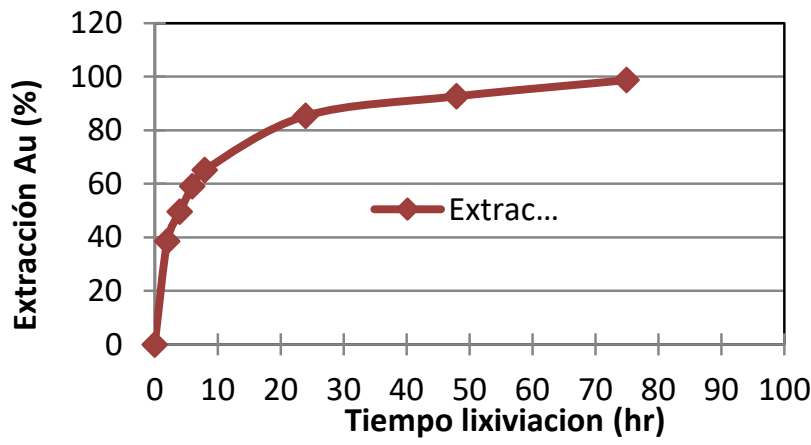

Figura 12. Cinética de Extracción Valores Metálicos Ag 0.3\% 
Analizando las Figuras $\mathrm{N}^{\circ} 13$ y 14 se observa que el crecimiento de la ley pregnant fue continuo y elevado en especial en las primeras mediciones, y que su grado de extracción tuvo un mayor acercamiento al $100 \%$ buscado, posiblemente causado por el mayor contenido del agente lixiviante durante el proceso.

En base a estos datos obtenidos se puede establecer una comparación entre ambas concentraciones trabajadas.

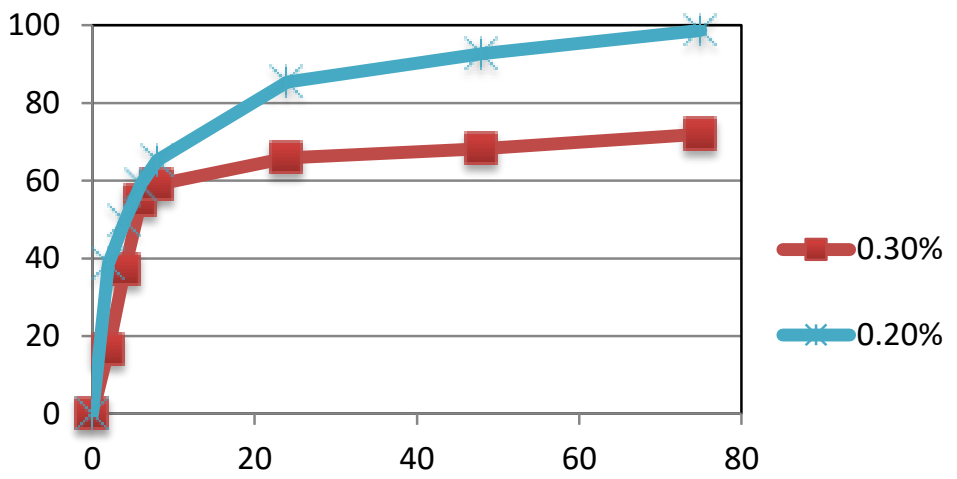

Figura 13. Comparación de la Cinética de Extracción de Plata al 0.2 y $0.3 \%$

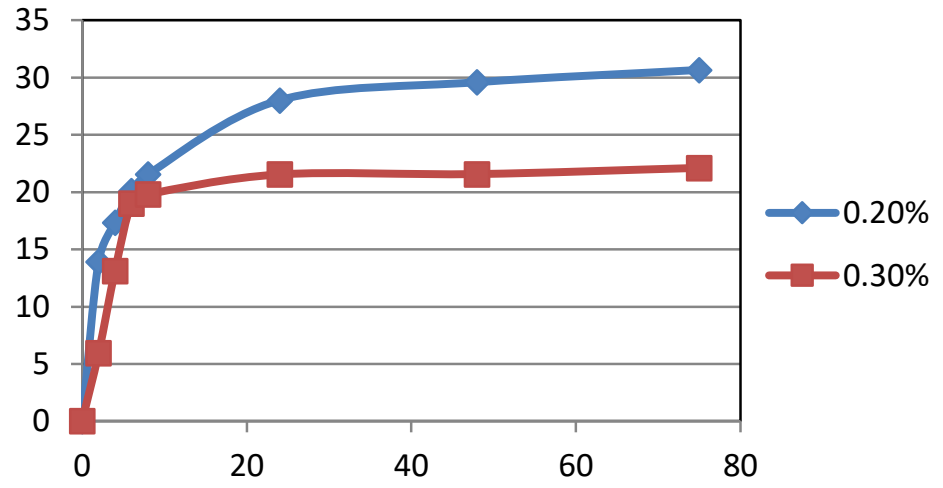

Figura 14. Comparación de Ley Pregnant al 0.2 y $0.3 \%$

De estas visualizamos que la concentración de Sandioss al 0.3\% tuvo una mayor cinética de extracción de finos de Plata en todo momento de la lixiviación, y que el crecimiento de la ley pregnant también fue mayor para la concentración de $0.3 \%$, dando a notar claramente que concentración resulta más óptima bajo las condiciones empleadas.

\section{CONCLUSIONES}

La utilización de Sandioss como agente lixiviante aun para bajas concentraciones resulto sumamente eficiente, en especial al aumentarle la concentración ya que se logró casi en su totalidad extraer la plata presente en finos.

El Sandioss si bien no tiende a alterar el $\mathrm{pH}$ de forma abrumado como si lo hace el cianuro una vez se ha consumido, suele arrojar a veces pHs mayores a los calculados en mediciones previas, esto se da a la naturaleza de sus componentes, ya que presenta feldespatos en baja 
concentración y otros elementos entre livianos y pesados, pero en su mayor cantidad básicos.

$\mathrm{Al}$ aumentar la concentración de Sandioss aumento la extracción en un 26.75\%, dándose a notar que para leyes bajas no es aconsejable trabajar con leyes muy bajas de Sandioss En las leyes de solución rica de 0.2 y $0.3 \%$ de Sandioss se observó que en las primeras horas la disolución es más rápida y al finalizar se vuelve más lenta, pero en especial en $0.2 \%$ notamos la baja crecida en la cinética de extracción, ello probablemente debido a que le falte mayor tiempo de lixiviación causado por el bajo contenido del agente lixiviante en la solución, sin embargo esto podría causar que diferentes elementos no valiosos reaccionen junto con los restos de Plata, lixiviando así por error componentes no deseados.

En la cinética de extracción de plata a 0.2 y $0.3 \%$ de Sandioss

- En la gráfica de concentración de $0.2 \%$ de Sandioss, se observa que a las 24 horas tenemos un $86.52 \%$ de la capacidad total (se da la lixiviación más fuerte)

- En la gráfica de concentración de $0.3 \%$ de Sandioss, se observa que a las 24 horas tenemos un $91.6 \%$ de la capacidad total (se da la lixiviación más fuerte)

\section{Agradecimientos}

Agradecemos al Vicerrectorado de Investigación y Posgrado - UNMSM por el financiamiento al proyecto $\mathrm{N}^{\circ} 161601021$, al Grupo de Investigación - TecMetS, a la Escuela Profesional de Ingeniería Metalúrgica de la UNMSM por permitirnos el uso de sus laboratorios y equipos, a la Empresa H\&F por su apoyo con los análisis de las muestras y a nuestro colaborador Anselmo Sánchez por brindarnos asesoramiento y ser un apoyo constante.

\section{Referencias}

Azañero Ortiz, Ángel. (1987). Informe técnico 951, LMC Banco Minero del Perú.

Habashi.F. (2006). Pressure Hydrometallurgy Update, XXII International Mineral Processing Congress, Istanbul.

Landauro, C. (2010). Informe de Caracterización de DRX, FRX y EM.

Lovera et al, 2017. Lixiviación de la Plata en minerales sulfurados argentíferos del Complejo Marañón con el reactivo Sandioss. VII Congreso Internacional Latinometalurgia, Cusco - Perú.

Lovera et al. (2016) Lixiviación. Extracción por solventes y Electro refinación de la plata a partir de minerales sulfurados argentíferos, Informe Final del Proyecto de Investigación $\mathrm{N}^{\circ}$ 161601021. VRI - IIGEO -UNMSM.

Lovera.D, Bustamante. A. (2005). Caracterización Fisicoquímica y Pruebas Metalúrgicas en la Prospección de Minerales Polimetálicos en el Complejo Marañón - Perú. Revista del IIGEO, Vol. 8, N 16: 44-50.

Luis A. Sánchez Quispe, Edwar W. Villavicencio Jaimes, Vladimir Arias A., Daniel Lovera D, Vidal S. Aramburú Rojas. (2013). Evaluación sistematizada y proceso metalúrgico para minerales sulfurados complejos de oro. Revista del Instituto de Investigación de la Facultad de Ingeniería Geológica, Minera, Metalúrgica y Geográfica. Vol. 16, Núm. 32.

Mantell, C. L. (1980) Ingeniería Electroquímica. Editorial Reverte, S. A. 
MINEM - http://www.minem.gob.pe/_estadistica.php?idSector=1\&idEstadistica=8639

Mirelly Araujo, Ángel Azañero, Daniel Lovera. (2014). Optimización en la recuperación de oro de minerales mixtos en Cerro Corona Goldfields La Cima. Revista del Instituto de Investigación de la Facultad de Ingeniería Geológica, Minera, Metalúrgica y Geográfica. Vol. 17, Núm. 33.

Misari CH., Fidel (1993). "Metalurgia Del Oro”, CEPECT, Lima-Perú, Tomo I y II.

Morales, M. P. (2010) Prueba Piloto de Aglomeración y Lixiviación en Montón, Proyecto La Quinua en Yanacocha. Ingeniería Metalúrgica. UNMSM.

Quiñones, J. (2009, 2010). Informe Microscopia óptica del mineral sulfurado del Complejo Marañon.

Vladimir Arias Arce, Rosa Coronado Falcón, Luis Puente Santibañez, Daniel Lovera Dávila. Refractariedad de concentrados auríferos. (2005). Revista del Instituto de Investigación de la Facultad de Ingeniería Geológica, Minera, Metalúrgica y Geográfica. Investigación de la Facultad de Ingeniería Geológica, Minera, Metalúrgica y Geográfica Vol. 8, Núm. 16. 